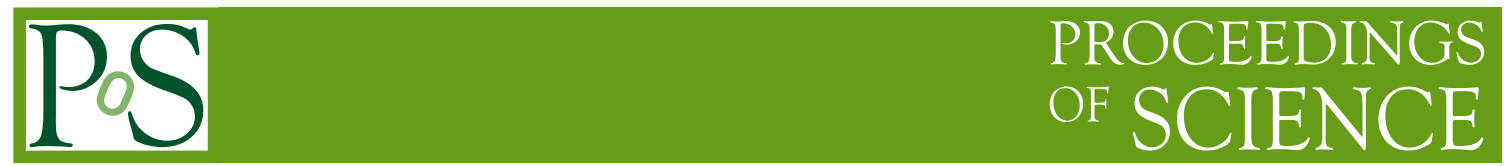

\title{
LHCb: Exotic Spectroscopy
}

\section{Lorenzo Capriotti ${ }^{a, *}$, on behalf of the LHCb collaboration}

${ }^{a}$ Alma Mater Studiorum Università di Bologna

INFN - Sezione di Bologna

E-mail: lorenzo.capriotti@unibo.it

Since the discovery of the $\chi_{c 1}$ (3872) meson in 2003, the field of exotic spectroscopy has thrived with the discovery of many multiquark candidates and the study of their properties, their production mechanisms and their decays, which provide a unique benchmark for effective theories of the strong interacion. Recent results from $\mathrm{LHCb}$ on exotic spectroscopy are presented, focussing on precise measurements of the already-established $\chi_{c 1}(3872)$ and the observation of new tetraquark candidates.

BEAUTY2020

21-24 September 2020

Kashiwa, Japan (online)

${ }^{*}$ Speaker 


\section{Introduction}

In 2003, the Belle collaboration reported the observation of a new charmonium-like state decaying into $J / \psi \pi^{+} \pi^{-}$[1]. Its mass, $3871.69 \mathrm{MeV} / c^{2}$, and its extremely narrow width - limited by the experimental resolution to be less than $1.2 \mathrm{MeV} / c^{2}$ - are incompatible with any predicted $c \bar{c}$ state. Given its strange properties, the newly discovered $\chi_{c 1}(3872)$ meson is labelled as exotic: due to the proximity to the $D^{0} \bar{D}^{* 0}$ mass threshold, it is believed to be a good candidate for a fourquarks bound state. Since then, more than 20 new exotic particles have been observed by different experiments, including the charged $Z_{c}$ states [2] and the pentaquark candidates [3], which further corroborate the multiquark interpretation of exotic particles. In the following, the latest results from the rich and productive exotic spectroscopy programme of the LHCb experiment are presented.

\section{Mass, width and lineshape of the $\chi_{c 1}(3872)$ state from inclusive $b$ decays}

The mass difference $m_{\chi_{c 1}(3872)}-m_{D^{0} \bar{D}^{* 0}}=0.01 \pm 0.18 \mathrm{MeV} / c^{2}$ [4] is compatible with zero; therefore, a precise measurement of the mass and width of the $\chi_{c 1}(3872)$ state is paramount in order to establish its nature (compact tetraquark, mesonic molecule or other binding mechanisms [5]). In order to do so, $3 \mathrm{fb}^{-1}$ of $p p$ collisions data collected by the LHCb experiment during Run 1 are analysed to reconstruct inclusive $\chi_{c 1}(3872) \rightarrow J / \psi \pi \pi$ from $b$ decays [6]. A simultaneous fit to the invariant mass spectra of 2011 and 2012 data divided in 3 bins of momentum is performed, using as signal shape either a relativistic Breit-Wigner or a Flatté-inspired model; the latter takes into account the $D^{0} \bar{D}^{* 0}$ threshold opening. The two models are found to be indistinguishable after a convolution with a resolution function, and the Breit-Wigner mass and width of the $\chi_{c 1}$ (3872) state are extracted from the fit:

$$
\begin{gathered}
m_{\chi_{c 1}(3872)}^{B W}=3871.695 \pm 0.067 \pm 0.068 \pm 0.010 \mathrm{MeV} / c^{2}, \\
\Gamma_{\chi_{c 1}(3872)}^{B W}=1.39 \pm 0.24 \pm 0.10 \mathrm{MeV} / c^{2},
\end{gathered}
$$

marking the first evidence for a nonzero Breit-Wigner width of the $\chi_{c 1}(3872)$ state. The last uncertainty on the mass measurement comes from the known uncertainty on the $\psi(2 S)$ mass, as the measurement is actually made of the mass difference between the $\chi_{c 1}(3872)$ and the $\psi(2 S)$ states. A study of the analytic structure of the decay amplitude near the $D^{0} \bar{D}^{* 0}$ threshold is also performed. The complex amplitude is a function of the square root of the energy; this gives rise to a two-sheeted Riemann surface in the complex plane. Using the Flatte amplidute model, two poles are found as shown in Fig.1: one in the physical sheet and one in the unphysical sheet. When the coupling of all channels except $D^{0} \bar{D}^{* 0}$ are sent to zero, the pole position moves towards the real axis below threshold, at $\operatorname{Re}(E)=-24 \mathrm{keV}$. This indicates a preference towards a bound-state description of the $\chi_{c 1}(3872)$, although a virtual assignment cannot be ruled out. If the state is interpreted as a bound state, its binding energy must be $E_{b}<100 \mathrm{keV}$, with a probability of finding a compact tetraquark component in its wavefunction of less than $33 \%$.

\section{3. $\psi_{2}(3283)$ and $\chi_{c 1}(3872)$ in $B^{+} \rightarrow J / \psi \pi^{+} \pi^{-} K^{+}$decays}

The $\psi_{2}$ (3823) state has been observed only in the $\chi_{c 1} \gamma$ final state with more than $5 \sigma$ [7]. A search for the $\psi_{2}$ (3283) decaying into $J / \psi \pi^{+} \pi^{-}$has been performed by the LHCb experiment 

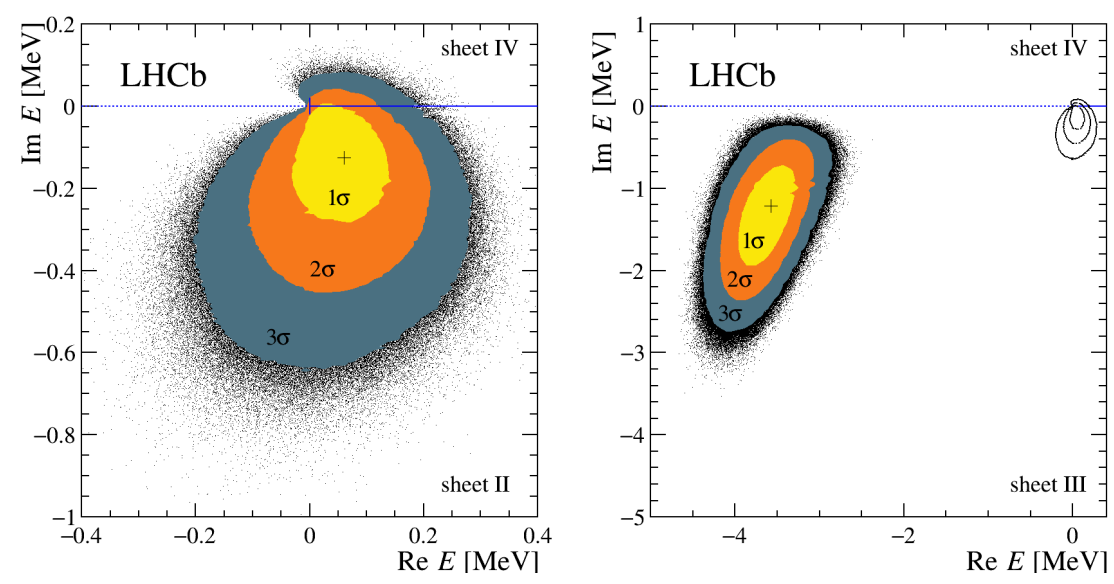

Figure 1: Poles of the $\chi_{c 1}(3872) \rightarrow J / \psi \pi \pi$ Flatté amplitude on the physical (left) and unphysical (right) sheets

using $9 \mathrm{fb}^{-1}$ of data collected in Runs 1 and 2, using both $\psi(2 S)$ and $\chi_{c 1}(3872)$ decaying in the same final state as normalisation channels; this allows not only the study of $\psi_{2}(3823)$, but also the measurement of the Breit-Wigner mass and width of the $\chi_{c 1}$ (3872). By performing bidimensional fits to $m_{B^{+}}$and $m_{J / \psi \pi \pi}$ for the three channels, shown in Fig.2, the $\psi_{2}(3823)$ is observed with a significance of $5.1 \sigma$ and several observables are measured:

$$
\begin{gathered}
\left.\frac{\mathcal{B}\left(B^{+} \rightarrow \psi_{2}(3823) K^{+}\right) \times \mathcal{B}\left(\psi_{2}(3823) \rightarrow J / \psi \pi^{+} \pi^{-}\right)}{\mathcal{B}\left(B^{+} \rightarrow \chi_{c 1}(3872) K^{+}\right) \times \mathcal{B}\left(\chi_{c 1}(3872) \rightarrow J / \psi \pi^{+} \pi^{-}\right)}=(3.56 \pm 0.67 \pm 0.11) \times 10^{-2}\right), \\
\left.\frac{\mathcal{B}\left(B^{+} \rightarrow \psi_{2}(3823) K^{+}\right) \times \mathcal{B}\left(\psi_{2}(3823) \rightarrow J / \psi \pi^{+} \pi^{-}\right)}{\mathcal{B}\left(B^{+} \rightarrow \psi(2 S) K^{+}\right) \times \mathcal{B}\left(\psi(2 S) \rightarrow J / \psi \pi^{+} \pi^{-}\right)}=(1.31 \pm 0.25 \pm 0.04) \times 10^{-3}\right), \\
\left.\frac{\mathcal{B}\left(B^{+} \rightarrow \chi_{c 1}(3872) K^{+}\right) \times \mathcal{B}\left(\chi_{c 1}(3872) \rightarrow J / \psi \pi^{+} \pi^{-}\right)}{\mathcal{B}\left(B^{+} \rightarrow \psi(2 S) K^{+}\right) \times \mathcal{B}\left(\psi(2 S) \rightarrow J / \psi \pi^{+} \pi^{-}\right)}=(3.69 \pm 0.07 \pm 0.06) \times 10^{-3}\right), \\
m_{\psi_{2}(3823)}=3824.08 \pm 0.53 \pm 0.14 \pm 0.01\left(m_{\psi(2 S)}\right) \mathrm{MeV} / c^{2}, \\
\Gamma_{\psi_{2}(3823)<5.2 \mathrm{MeV} / c^{2},} \\
m_{\chi_{c 1}(3872)}=3871.59 \pm 0.06 \pm 0.03 \pm 0.01\left(m_{\psi(2 S)}\right) \mathrm{MeV} / c^{2}, \\
\Gamma_{\chi c 1}(3872)=0.96_{-0.18}^{+0.19} \pm 0.21 \mathrm{MeV} / c^{2},
\end{gathered}
$$

where $(1,2)$ are first measurements, $(3,4,6)$ are the most precise measurements, (5) is the world best limit and (7) confirms the nonzero width of the $\chi_{c 1}$ (3872) as shown in Section 2. Combining these latest $\mathrm{LHCb}$ measurements of the mass and width of the $\chi_{c 1}(3872)$ with previous ones, the new world averages are $m_{\chi_{c 1}(3872)}=3871.64 \pm 0.06 \mathrm{MeV} / c^{2}$ and $\Gamma_{\chi_{c 1}(3872)}=1.19 \pm 0.19 \mathrm{MeV} / c^{2}$.

\section{Observation of a structure in the $J / \psi$-pair invariant mass}

Several theoretical predictions place a hypothetical four-charm bound state in the mass range $[5.8,7.4] \mathrm{GeV} / \mathrm{c}^{2}$ (see Ref. [9] for a list of papers). The LHCb collaboration has analysed $9 \mathrm{fb}^{-1}$ of 

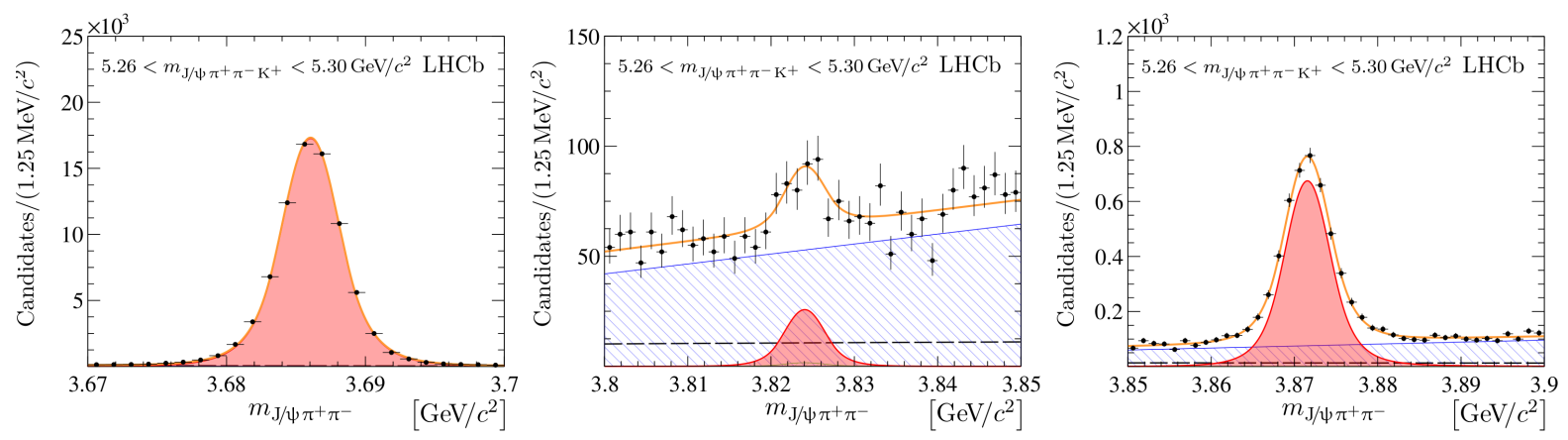

Figure 2: Fit projections to $m_{J / \psi \pi \pi}$ for the $\psi(2 S)$ (left), $\psi_{2}(3823)$ (center) and $\chi_{c 1}(3872)$ (right) peaks
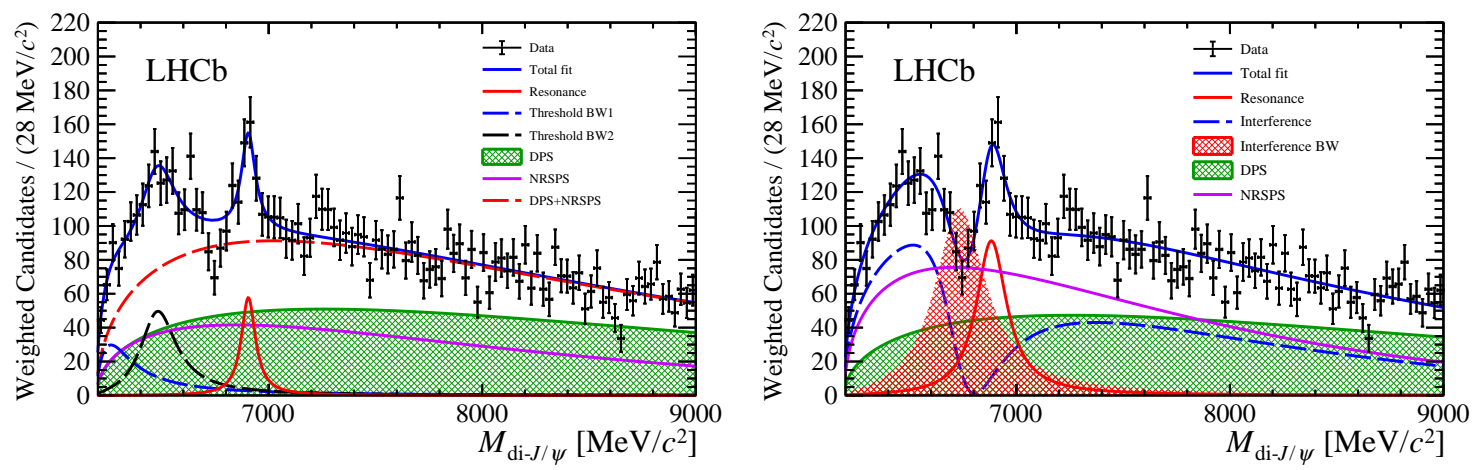

Figure 3: Fit to the $J / \psi$ pair invariant mass, where the threshold enhancement is described by two BreitWigners (left) or a single Breit-Wigner interfering with the NRSPS background (right)

$p p$ collision data collected during Runs 1 and 2 in order to search for a structure in that mass range, which is far away from any known resonating charmonia or bottomonia contribution. The invariant mass spectrum of four muons coming from two $J / \psi$ mesons, produced in the $p p$ interaction point, is studied. Two irreducible sources of background are considered: the double-parton scattering (DPS), i.e. a $J / \psi$ pair produced from two different partonic interactions; the non-resonant singleparton scattering (NRSPS), i.e. a $J / \psi$ pair produced through a single partonic interaction with no intermediate resonance. A threshold enhancement is clearly visible in data and it can be explained by adding two Breit-Wigner shapes, or a single Breit-Wigner interfering with the NRSPS. In both cases, a structure consistent with being a resonance is observed with a mass of about $6900 \mathrm{MeV} / \mathrm{c}^{2}$ and with a significance of more than $5 \sigma$; the fits are shown in Fig. 3. Further studies are required to investigate the nature of such structure, but if it is interpreted as a resonance it would be the first observation of an exotic hadron entirely composed by heavy quarks of the same flavour.

\section{Observation of the $\mathrm{X}(2900)$ state in $B^{+} \rightarrow D^{+} D^{-} K^{+}$decays}

The LHCb collaboration has analysed $B^{+} \rightarrow D^{+} D^{-} K^{+}$decays in order to search for exotic contributions, using the full Run 1 and Run 2 dataset. A first model-independent study [10] describes the rich resonant structure of the $D^{+} D^{-}$system by expanding its helicity angle in terms of Legendre polynomials, including Legendre moments from resonances up to $J=2$. As shown 

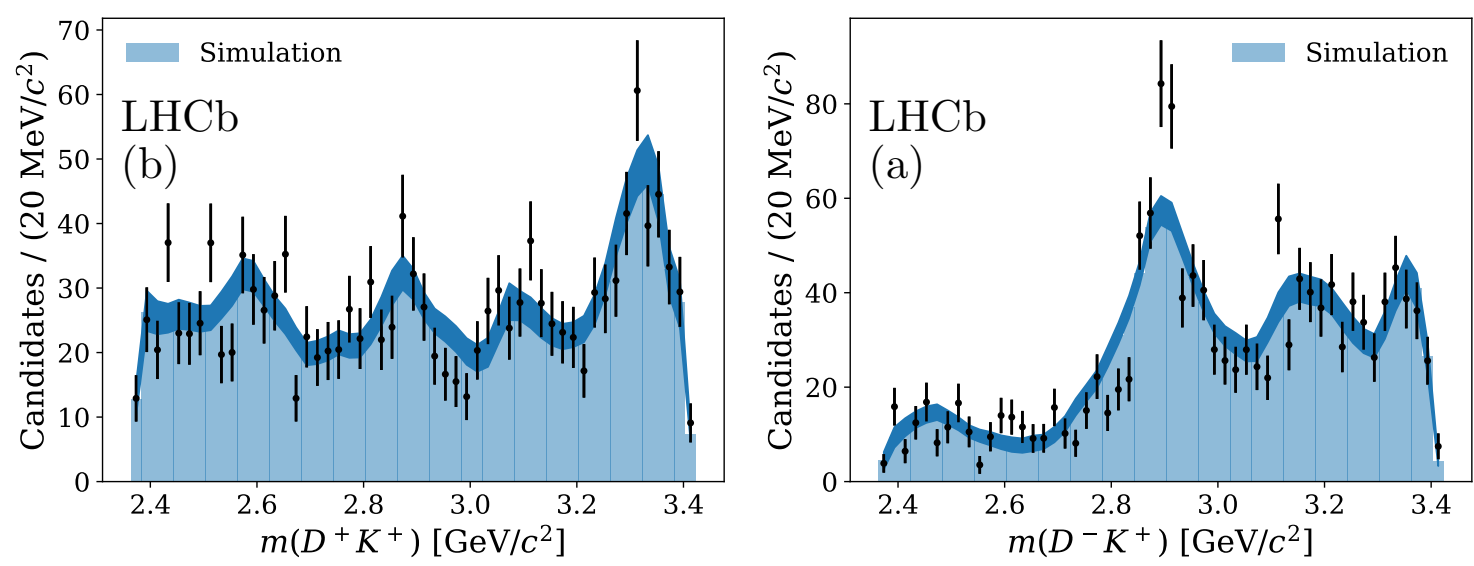

Figure 4: Invariant masses of the $D^{+} K^{+}$(left) and $D^{-} K^{+}$(right) systems from $B^{+} \rightarrow D^{+} D^{-} K^{+}$decays, with Legendre polynomial expansion up to $J=2$ overlaid.

in Fig. 4, the model explains satisfactorily the $D^{+} K^{+}$mass spectrum but an unexplained structure is present in the $D^{-} K^{+}$mass spectrum; the hypothesis that the latter can be explained using only $D^{+} D^{-}$resonances up to spin 2 is rejected with a significance of $3.9 \sigma$. In order to investigate further, an amplitude analysis is performed [11]. The amplitude model is built with the isobar formalism, using relativistic Breit-Wigners with Zemach tensors and Blatt-Weisskopf barrier factor for all the well-motivated and known $D^{+} D^{-}$resonances. Data are found to be well described by the model, as shown in Fig. 5, if two additional $D^{-} K^{+}$Breit-Wigners are included, one scalar and one vector with roughly the same mass:

$$
\begin{gathered}
m_{X_{0}(2900)}=2886 \pm 7 \pm 2 \mathrm{MeV} / c^{2}, \Gamma_{X_{0}(2900)}=57 \pm 12 \pm 4 \mathrm{MeV} / c^{2}, \\
m_{X_{1}(2900)}=2904 \pm 5 \pm 1 \mathrm{MeV} / c^{2}, \Gamma_{X_{1}(2900)}=110 \pm 11 \pm 4 \mathrm{MeV} / c^{2} .
\end{gathered}
$$

Furthermore, no evidence for the $\chi_{c 0}(3860) \rightarrow D^{+} D^{-}$state reported by Belle is found, and the $\chi_{c 2}(3930)$ contribution is better described by splitting it in two states:

$$
\begin{aligned}
& m_{\chi_{c 0}(3930)}=3923.8 \pm 1.5 \pm 0.4 \mathrm{MeV} / c^{2}, \Gamma_{\chi_{c 0}(3930)}=17.4 \pm 5.1 \pm 0.8 \mathrm{MeV} / c^{2} \\
& m_{\chi_{c 2}(3930)}=3926.8 \pm 2.4 \pm 0.8 \mathrm{MeV} / c^{2}, \Gamma_{\chi_{c 2}(3930)}=34.2 \pm 6.6 \pm 1.1 \mathrm{MeV} / c^{2} .
\end{aligned}
$$

Regarding the $X(2900)$ states, if interpreted as resonances, they would indicate the first clear observation of exotic hadrons with open flavour, with minimal quark content $[c d \bar{s} \bar{u}]$, although other models - for instance rescattering - may also explain the discrepancy.

\section{References}

[1] S. Choi et al. (Belle collaboration), Observation of a Narrow Charmoniumlike State in Exclusive $B^{ \pm} \rightarrow K^{ \pm} \pi^{+} \pi^{-} J / \psi$ Decays, Phys. Rev. Lett. 91 (2003) 262001.

[2] N. Brambilla et al., The XYZ states: experimental and theoretical status and perspectives, Physics Reports 873 (2020) 1-154 [hep-ex/1907.07583]. 

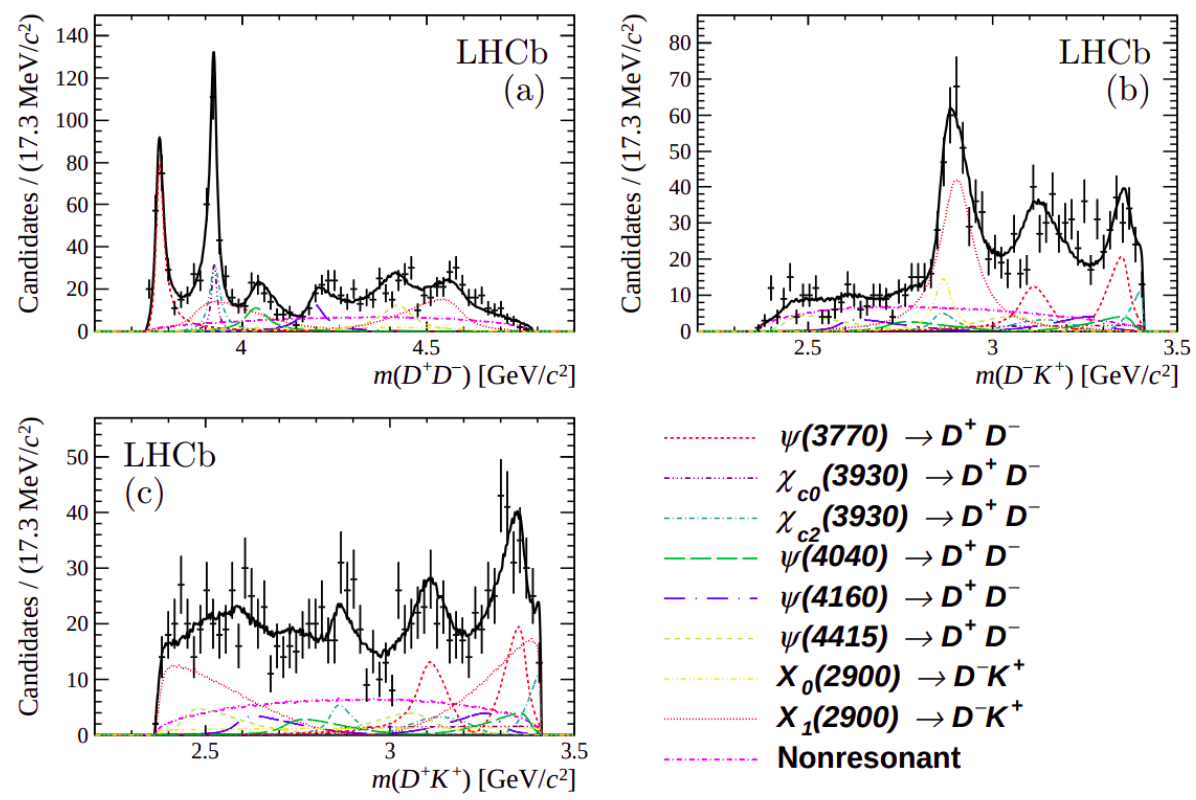

Figure 5: Amplitude analysis of the $B^{+} \rightarrow D^{+} D^{-} K^{+}$decay, with the two additional exotic contributions and the splitting of $\chi_{c 0}(3930)$.

[3] R. Aaij et al. (LHCb collaboration), Observation of $J / \psi p$ Resonances Consistent with Pentaquark States in $\Lambda_{b}^{0} \rightarrow J / \psi K^{-}$p Decays, Phys. Rev. Lett. 115 (2015) 072001.

[4] P.A. Zyla et al. (Particle Data Group), Prog. Theor. Exp. Phys. 2020, 083 C01 (2020).

[5] S. L. Olsen et al., Nonstandard heavy mesons and baryons: Experimental evidence, Rev. Mod. Phys. 90 (2018) 015003.

[6] R. Aaij et al. (LHCb collaboration), Study of the lineshape of the $\chi_{c 1}(3872)$ state, Phys. Rev. D 102 (2020) 092005.

[7] M. Ablikim et al. (BESIII collaboration), Observation of the $\psi\left(1^{3} D_{2}\right)$ State in $e^{+} e^{-} \rightarrow$ $\pi^{+} \pi^{-} \gamma \chi_{c 1}$ at BESIII, Phys. Rev. Lett. 115 (2015) 011803.

[8] R. Aaij et al. (LHCb collaboration), Study of the $\psi_{2}(3823)$ and $\chi_{c 1}(3872)$ states in $B^{+} \rightarrow$ $\left(J / \psi \pi^{+} \pi^{-}\right) K^{+}$decays, JHEP 08 (2020) 123.

[9] R. Aaij et al. (LHCb collaboration), Observation of structure in the J/4-pair mass spectrum, Sci. Bull. 65 (2020) 1983.

[10] R. Aaij et al. (LHCb collaboration), Model-independent study of structure in $B^{+} \rightarrow D^{+} D^{-} K^{+}$ decays, Phys. Rev. Lett. 125 (2020) 242001.

[11] R. Aaij et al. (LHCb collaboration), Amplitude analysis of the $B^{+} \rightarrow D^{+} D^{-} K^{+}$decay, Phys. Rev. Lett. 102 (2020) 112003. 